Las ORT parecen ser hallazgos frecuentes en la SD-OCT de distrofias maculares con cambios atróficos de la citoarquitectura de la retina externa pudiendo constituir un evento tardío de la degeneración retiniana, fuertemente asociado a la disrupción de la MLE. Nuevos estudios con un mayor número de pacientes deben ser realizados para evaluar la utilidad de este hallazgo en el seguimiento y evaluación pronóstica de estos pacientes, así como su posible uso en la monitorización del efecto neuroprotector de nuevas terapias en desarrollo.

B I B L I O G R A F Í A

1. Zweifel SA, Engelbert M, Laud K, Margolis R, Spaide RF, Freund KB. Outer retinal tubulation: a novel optical coherence tomography finding. Arch Ophthalmol. 2009;127:1596-602.

2. Cohen SY, Dubois L, Nghiem-Buffet S, et al. Retinal pseudocysts in age-related geographic atrophy. Am J Ophthalmol. 2010;150:211-7.

R. Dolz-Marco a,*, R. Gallego-Pinazo a , M.D. Pinazo-Durán ${ }^{\text {b,c }}$, J.F. Arévalo ${ }^{\text {d,e }}$, L.A. Yannuzzi ${ }^{\mathrm{f}}$ y M. Díaz-Llopis ${ }^{\mathrm{a}, \mathrm{c}}$ a Department of Ophthalmology, University and Polytechnic Hospital La Fe, Valencia, España

b Opthalmology Research Unit Santiago Grisolía, University Hospital Doctor Peset. Valencia, Spain

${ }^{c}$ Faculty of Medicine, University of Valencia, Valencia, Spain

$\mathrm{d}$ Retina Division, Wilmer Eye Institute, Johns Hopkins University

School of Medicine, Baltimore, MD, USA

e King Khaled Eye Specialist Hospital, Riyadh, Kingdom of Saudi

Arabia

${ }^{f}$ VitreousRetinal Macula Consultants of New York. LuEsther T.

Mertz Retinal Research Center, Manhattan Eye, Ear and Throat

Hospital, New York, USA

* Autor para correspondencia.

Correo electrónico: rosadolzmarco@gmail.com

(R. Dolz-Marco).

0365-6691/\$ - see front matter

(C) 2012 Sociedad Española de Oftalmología. Publicado por

Elsevier España, S.L. Todos los derechos reservados.

http://dx.doi.org/10.1016/j.oftal.2012.02.009

\title{
Uso de cuestionarios de calidad de vida para la evaluación de pacientes sometidos a cirugía de catarata
}

\section{Use of quality of life questionnaires for the evaluation of patients subjected to cataract surgery}

\section{Sr. Director:}

Durante las últimas décadas se ha incrementado el uso de cuestionarios de calidad de vida como instrumentos basados en el reporte del paciente ${ }^{1}$. Sin embargo, estos cuestionarios han sido utilizados principalmente en el campo de la investigación, mientras que su aplicación en la práctica médica oftalmológica se encuentra constantemente ignorada. $t$

En la evaluación del paciente post-operado, convencionalmente, se mide la agudeza visual, hallazgos en la biomicroscopía, presencia de nuevos síntomas, entre otros. Los cuestionarios de calidad de vida proporcionan una herramienta adicional que permite obtener una evaluación que incluye la perspectiva del paciente con respecto a su estado funcional y satisfacción relacionada con su visión después del tratamiento quirúrgico. Para lograr una evaluación integral del paciente, es importante considerar este aspecto. Por otro lado, es también importante tener en cuenta los costos y el tiempo que implicaría la aplicación de estos cuestionarios; sin embargo, es la mejor forma de evaluar la respuesta terapéutica y evitar ciertas discordancias que se puedan presentar entre la toma de agudeza visual y la discapacidad visual del paciente ${ }^{2}$.

El beneficio de estos instrumentos depende de su correcto uso. Para elegir el más apropiado, es importante conocer que existen instrumentos que evalúan de manera más específica la calidad de vida relacionada con el estado de salud ocular y con enfermedades oculares específicas como cataratas (tabla 1). Lundstrom y Pseudovs ${ }^{1}$ analizan las propiedades psicométricas de algunos cuestionarios de calidad de vida usados para pacientes con cataratas y concluyen que existen detalles que influyen en la utilidad de los cuestionarios en una población dada, como el grado de discapacidad promedio generado por las cataratas en los pacientes al momento de decidir operarse, el grado de educación promedio de los

Tabla 1 - Principales características de 4 instrumentos para medir calidad de vida en oftalmología

\begin{tabular}{lcccc} 
& $\begin{array}{c}\text { Idioma } \\
\text { original }\end{array}$ & $\begin{array}{c}\text { Número de } \\
\text { preguntas }\end{array}$ & $\begin{array}{c}\text { Validación al Cronbach } \\
\text { español }\end{array}$ \\
\hline ADVS & Inglés & 22 & No & $\alpha \geq 0,90$ \\
Cataract TyPE & Inglés & 12 & No & $\alpha \geq 0,94$ \\
$\quad$ Specification & & & & \\
$\quad$ Questionnaire & & & & \\
Catquest & Inglés & 19 & No & $\alpha \geq 0,93$ \\
NEI VFQ 25 & Inglés & 25 & Sí & $\alpha \geq 0,86$ \\
\hline
\end{tabular}

ADVS: Activities of Daily Vision Scale; NEI VFQ-25: National Eye Institute Visual Function Questionnaire; VF-14: Visual Function-14. Fuente: Basada de Lundström et al. ${ }^{1}$ 
pacientes, diferencias culturales y otras características particulares de cada población.

Para definir el momento de aplicación del cuestionario de calidad de vida después de la cirugía de cataratas, Limburg et $\mathrm{al}^{3}$. observan que el grupo de pacientes que presentan pobre respuesta en las primeras semanas, después de la cirugía, tienden a alcanzar su nivel más óptimo de visión entre los 4 y 6 meses. El momento más oportuno para la aplicación del cuestionario debería ser en el intervalo de las 12 semanas después de la cirugía, que es el tiempo promedio de seguimiento recomendado después de la operación.

En conclusión, es necesario promover el uso correcto y cuidadoso de cuestionarios de calidad de vida en pacientes sometidos a la cirugía de cataratas, considerando las características de la población a evaluar, lo cual permitirá al médico tratante conseguir una evaluación integral del paciente.

\section{B I B L I O G R A F Í A}

1. Lundström M, Pesudovs K. Questionnaires for measuring cataract surgery outcomes. J Cataract Refract Surg. 2011;37:945-59.
2. Cabezas M, Gracia J, García J, Morente P. Calidad de vida en pacientes intervenidos de catarata. Arch Soc Esp Oftalmol. 2005;80:449-56.

3. Limburg H, Foster A, Gilbert C, Johnson GJ, Kyndt M, Myalt M. Routine monitoring of visual outcome of cataract surgery. Part 2: Results from eight study centres. Br J Ophthalmol. 2005;89:50-2.

\section{S. Luján*, M. Alburquerque y O. Pizango}

Escuela de Medicina, Universidad Peruana de Ciencias Aplicadas, Lima, Perú

* Autor para correspondencia.

Correo electrónico: si_lujan@yahoo.com (S. Luján).

0365-6691/\$ - see front matter

(c) 2012 Sociedad Española de Oftalmología. Publicado por Elsevier España, S.L. Todos los derechos reservados. http://dx.doi.org/10.1016/j.oftal.2012.04.015 